\title{
Application of high-temperature superconducting permanent magnets to synch rotron radiation sources
}

\author{
Takashi Tanaka, ${ }^{*}$ Toru Hara, and Hideo Kitamura \\ RIKEN/SPring-8, Koto 1-1-1, Mikazuki, Sayo, Hyogo 679-5148, Japan \\ Rieko Tsuru \\ School of Science, University of Hyogo, Kouto 3-2-1, Kamigori, Ako, Hyogo 678-1297, Japan \\ Teruhiko Bizen, Xavier Maréchal, and Takamitsu Seike \\ JASRI/SPring-8, Koto 1-1-1, Mikazuki, Sayo, Hyogo 679-5198, Japan
}

(Received 12 July 2004; published 29 September 2004)

\begin{abstract}
A simple scheme for field enhancement in synchrotron radiation sources such as undulators and wigglers is proposed, which is based on the fundamental nature of the superconducting loop where the magnetic flux is preserved. A superconductor ring placed to enclose the magnetic pole works as a kind of permanent magnet. The magnetization is performed by electromagnetic induction brought by the opening movement of the magnetic gap. Since neither additional external power supplies nor current leads are necessary, high-temperature bulk superconductors can easily be implemented in this scheme. Calculations to check the effectiveness of the new concept show that the critical current density of the superconductor is crucial to the performance of the synchrotron radiation sources based on this concept. Experiments were performed to verify the principle of the proposed scheme, which gave promising results to strongly support it.
\end{abstract}

DOI: 10.1103/PhysRevSTAB.7.090704

PACS numbers: 41.60.Ap, 84.71.Ba

\section{INTRODUCTION}

Permanent magnet (PM) synchrotron radiation (SR) sources such as undulators [1] have been utilized in many SR facilities [2-4] to generate quasimonochromatic intense SR. The current trend in undulator developments is toward shortening the magnetic period to obtain SR with shorter wavelength [5-8]. Therefore, much effort has been made to develop short period undulators such as an in-vacuum undulator $[9,10]$, a superconducting undulator (SCU) working around liquid helium temperature $[11,12]$, and a cryogenic permanent magnet undulator (cryoundulator) [13]. The last one has been recently proposed at SPring-8, which utilizes PMs under cryogenic environment.

The development of the SCU is still in progress since there are lots of technological problems, particularly, a thermal budget problem. On the other hand, the cryoundulator will be easily realized because its principle is simple: just to reduce the temperature of the permanent magnets around liquid nitrogen temperature where the thermal budget problem is not so serious compared to that in SCUs. This undulator design causes two important effects on the PM material. One is to increase the remanent field $\left(B_{r}\right)$ by $10 \%-20 \%$, resulting in a higher peak field $\left(B_{p}\right)$ of the periodic field in the undulator. The other is to increase the coercivity at least twice, which improves significantly the resistivity against demagnetiza-

*Electronic address: ztanaka@spring8.or.jp tion due to electron beam irradiation during operation [14]. And furthermore, the cryoundulator concept gives us a new possibility to enhance the magnetic field by means of exploiting superconducting (SC) permanent magnets, i.e., the superconductors magnetized by a certain method. As is discussed later, high-temperature SC material [15-19] such as Y-Ba-Cu-O can be used for SC permanent magnets in the undulator based on this new concept, which allows us to operate the undulator at the temperature much higher than that of liquid helium, for example, $40-80 \mathrm{~K}$.

\section{PRINCIPLE}

In order to explain the principle of the cryoundulator with SC permanent magnets, let us compare it to a simple scheme for field enhancement in electromagnets, which is based on the fundamental nature of the SC loop where the magnetic flux is preserved. Figure 1(a) shows a magnetic circuit composed of a SC loop and an electromagnet with a pair of variable gaps. For convenience, we may assume that the cross section of the flux path is constant at any position and either the fringe effect or the flux leakage is negligible. In the case where the temperature of the SC loop is higher than the critical temperature $\left(T_{c}\right)$, the magnetic field $(B)$ at the gap is obtained by the simple Ampere's law as

$$
B=\frac{\mu_{0} N I}{2 g+l / k_{m}},
$$

C 2004 The American Physical Society

090704-1 

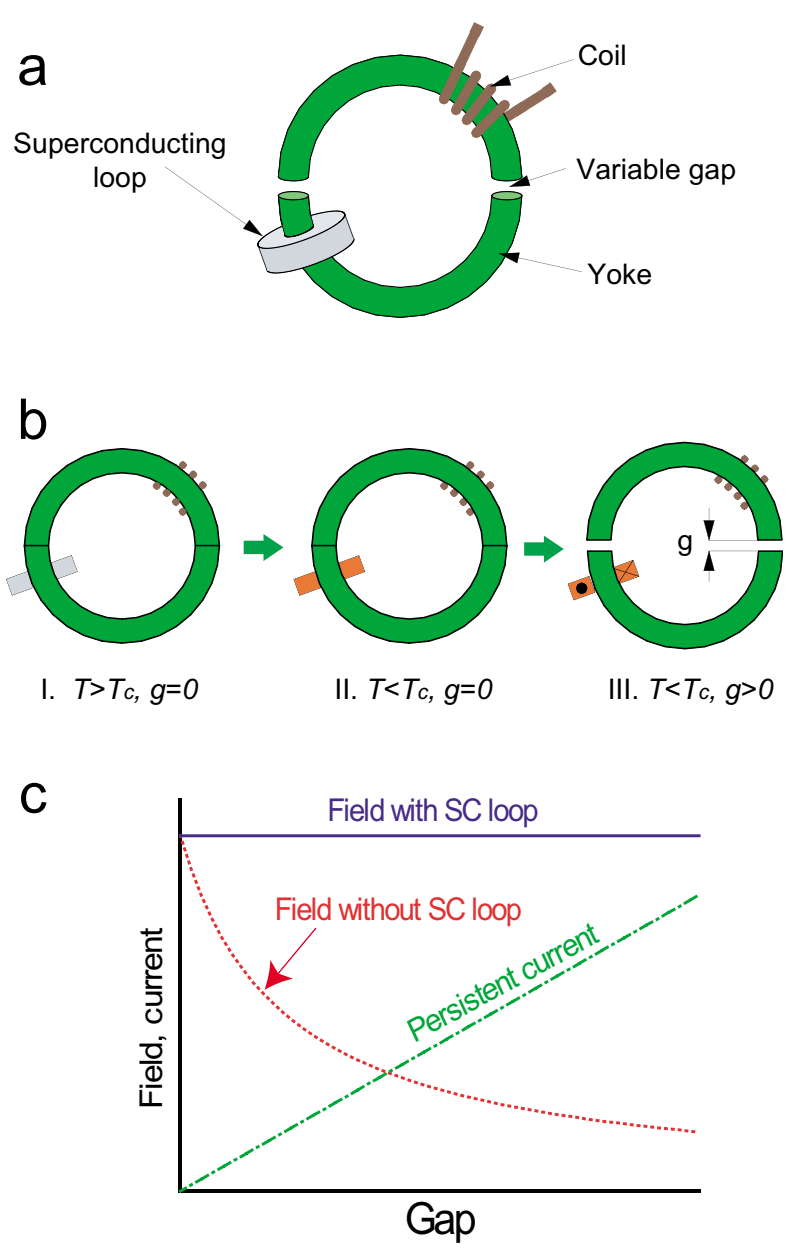

FIG. 1. (Color) Scheme of field enhancement in an electromagnetic circuit. (a) Schematic illustration of the magnetic circuit comprised of a SC loop and an electromagnet with a pair of variable gaps and (b) its magnetization process. (c) Typical gap dependence of the magnetic field at the gap without and with the superconductor as denoted by dotted and solid curves, respectively. Note that either the fringe effect or the flux leakage is assumed to be negligible.

where $N I$ is the magnetomotive force of the coil, $\mu_{0}$ the permeability of vacuum, $k_{m}$ the relative permeability of the yoke, $g$ the gap width, and $l$ the total length of the flux path in the yoke. The $k_{m}$ value typically ranges from 1000 to 10000 or higher if the yoke is not saturated. For example, permendur made from cobalt-iron-vanadium has a high $k_{m}$ value of about 1700 at the flux density of $2 \mathrm{~T}$ [20]. Therefore, we can expect an extremely high magnetic field at the fully closed gap, i.e., $g=0$. However, the magnetic field decreases drastically by opening the gap as denoted by a dotted curve in Fig. 1(c).

Now let us consider the case exploiting the superconductor. The SC loop can be magnetized according to the process shown in Fig. 1(b). At first the gap is fully closed at a temperature higher than the critical temperature $\left(T_{c}\right)(\mathrm{I})$. Then the superconductor is cooled to a tempera- ture lower than $T_{c}$ (II). When the gap is opened to ensure a space for application (III), an additional magnetomotive force is generated by the persistent current in the superconductor. Thus, the magnetic field in this case will keep its maximum value independently of the gap width as denoted by a solid curve in Fig. 1(c), where the persistent current is also shown schematically as a function of the gap width as denoted by a chain curve. Accordingly, we can expect a much higher field in the magnetic devices having air/vacuum gaps by exploiting the superconductors.

The above principle is easily applied to a PM device having a variable gap system. The undulator as an SR source is an important candidate because it usually has a gap-driving system to control the field for tuning the wavelength of SR. In particular, the cryoundulator operating around liquid nitrogen temperature is well suited to this application because it has a vacuum structure to cool the magnetic components inside and a mechanical structure to enable the fully closed gap. Let us call an undulator based on this concept a "cryoundulator plus" (CU plus), where bulk-type or multilayer-type hightemperature superconductors are expected to play an important role.

\section{EXAMPLE}

Let us estimate the achievable performance of CU plus using a model with the periodic length of $14 \mathrm{~mm}$, comprised of PMs and magnetic pole pieces, as shown in Figs. 2(a) and 2(b). The pole pieces are sandwiched by permanent magnets, and high-temperature superconductors (HTSCs) are put on the permanent magnets. As the PM and pole-piece materials, NEOMAX-53CR and Permendur (NEOMAX Co., Ltd) are assumed, respectively. NEOMAX-53CR, made from praseodymiumiron-boron, has the remanent field of $1.5 \mathrm{~T}$ at $77 \mathrm{~K}$ [13], and Permendur has the saturation magnetic flux density of $2.39 \mathrm{~T}$ [20]. Note that the pole length $\left(L_{p}\right)$ and the thickness of the superconductor $\left(T_{s}\right)$ determine the volume of the fraction of the permanent magnet to be removed. Therefore, the optimum values for these two parameters depend on the critical current density $\left(J_{c}\right)$ of the superconductor. The calculation for the achievable peak field was made taking the above condition into account.

The periodic magnetic field in the present $\mathrm{CU}$ plus model is calculated with RADIA [21], a three-dimensional magnetostatics computer code. In the calculation, the persistent current $\left(I_{p}\right)$ flowing in the superconductor is determined so that the magnetic flux enclosed by the superconductor ring is preserved at any gap with the initial condition that $I_{p}=0$ at the fully closed gap. Figure 2(c) shows the result of the calculation for two cases of the minimum gap ( 5 and $3 \mathrm{~mm}$ ) allowed for the electron beam to go through the undulator. We find a 

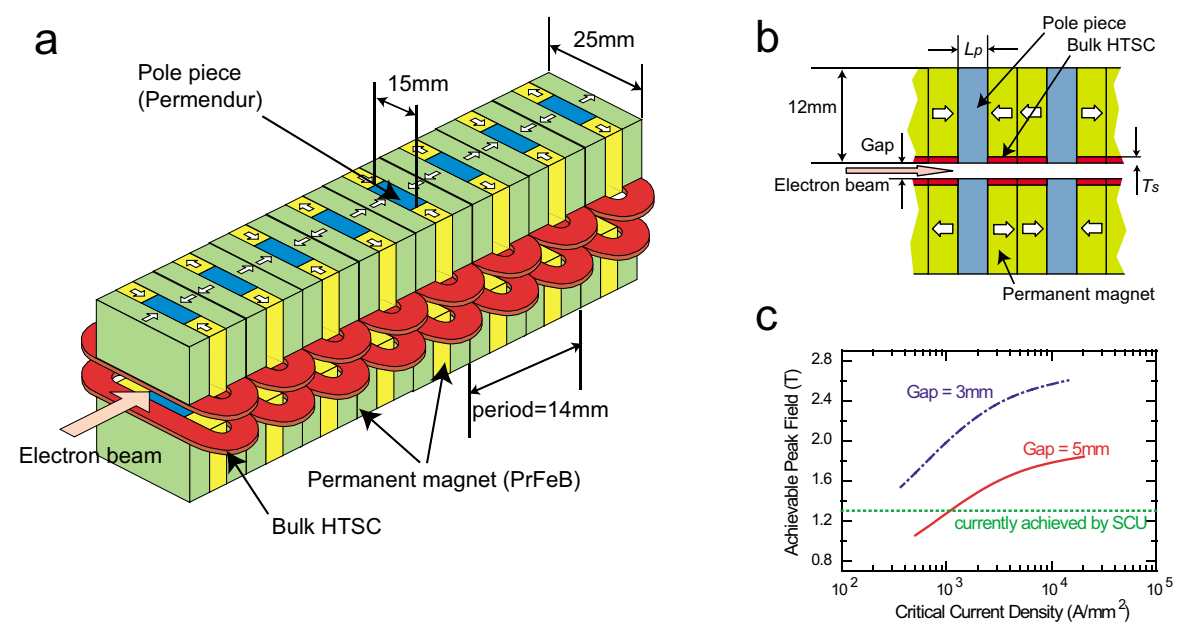

FIG. 2. (Color) Example of a magnetic structure model for cryoundulator (CU) plus (a),(b) and its magnetic performance (c). Detailed dimensions for each component are indicated in (a) and a longitudinal cross-sectional view of the CU plus model is given in (b). Achievable peak fields calculated as a function of $J_{c}$ are shown in (c) for two different values of the gap width, $5 \mathrm{~mm}$ (solid curve) and $3 \mathrm{~mm}$ (chain curve), respectively.

remarkable enhancement of the achievable $B_{p}$ as the critical current density $\left(J_{c}\right.$, the maximum current density which can flow in the superconductor) increases. If the superconductor has a $J_{c}$ value higher than $1.1 \mathrm{kA} / \mathrm{mm}^{2}$, $B_{p}$ of CU plus is expected to exceed that of the recent SCU [22] at the same gap.

It should be noted that the minimum gap of CU plus can probably be made narrower than that of the SCU operating around liquid helium temperature, because the operating temperature, $40-80 \mathrm{~K}$, of $\mathrm{CU}$ plus is much higher than that of liquid helium, which ensures a high cooling capacity of the state-of-the-art cryocoolers. In such a case, the performance of CU plus is further improved as indicated by a chain curve for gap $=3 \mathrm{~mm}$.

\section{EXPERIMENTS}

We performed experiments to verify the principle of $\mathrm{CU}$ plus with superconductor rings made from a commercially available material, $\mathrm{Gd}-\mathrm{Ba}-\mathrm{Cu}-\mathrm{O}$.

First we investigated the performance of the superconductor ring itself by applying an external field using a separated magnetic flux generator. The illustration and dimensions of the superconductor ring are shown in Fig. 3(a) and the results are in Fig. 3(b). The abscissa shows the strength of the external field, while the ordinate shows the field measured by a Hall probe at the center of the superconductor ring. The measurement was performed at the temperature of liquid nitrogen $(77 \mathrm{~K})$. We can find a typical hysteresis curve brought by the diamagnetism of the superconductor ring. From the experimental results, $J_{c}$ was roughly estimated at $\sim 200 \mathrm{~A} / \mathrm{mm}^{2}$ by comparison with a calculation of the magnetic field by a current loop with the same shape and dimensions as the superconductor ring. This value is indeed typical for a copper-oxide SC material [15].

Then we performed experiments using the test magnet assembly comprised of PMs and the superconductors, as shown in Fig. 4(a). The PMs were made from NEOMAX

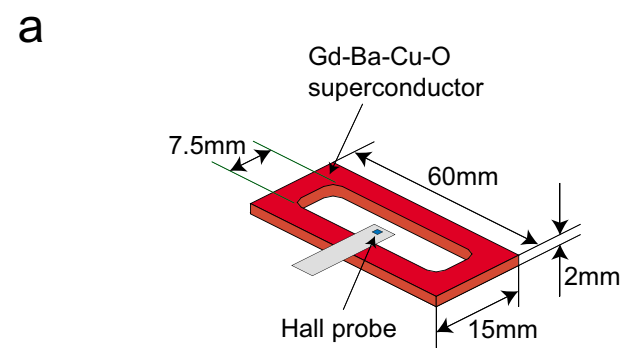

b

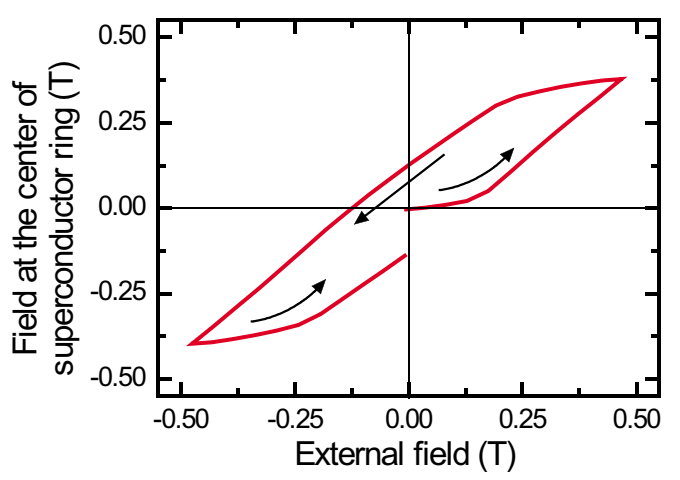

FIG. 3. (Color) Determination of the critical current density $\left(J_{c}\right)$ of the superconductor ring used for the test magnet assembly. (a) Illustration and dimensions of the superconductor ring and (b) result of the field measurement for the superconductor ring at the temperature of $77 \mathrm{~K}$. 

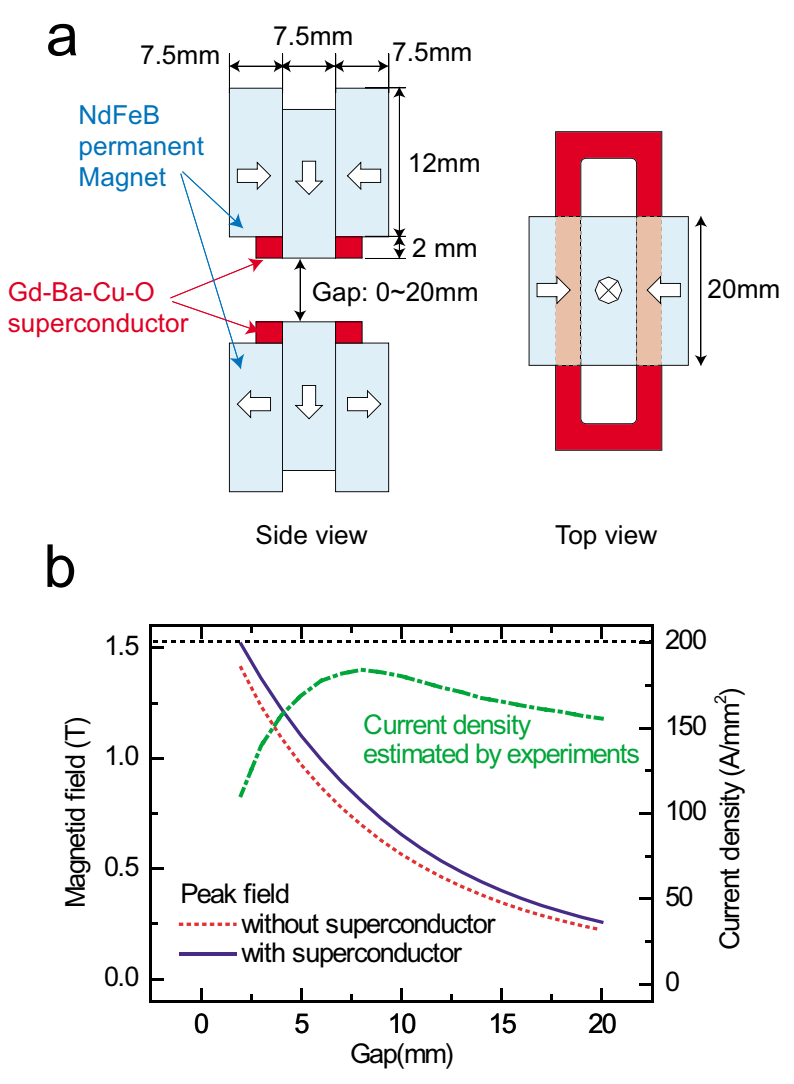

FIG. 4. (Color) Verification of the principle of cryoundulator plus. (a) Experimental setup of the test magnet assembly. The permanent magnets made from $\mathrm{NdFeB}$ were arranged according to the Halbach configuration and the superconductor rings were put on the permanent magnets. (b) Magnetic fields with and without the superconductors measured at the gap center as a function of the gap width. The chain curve shows the estimated current density in the superconductor.

50BH (NEOMAX Co., Ltd.) and arranged according to the pure Halbach configuration [23] except that the horizontally magnetized blocks were shifted upward or downward by $2 \mathrm{~mm}$ to insert the superconductors. Before starting the field measurement, we fully closed the gap at the temperature of $300 \mathrm{~K}$, cooled the assembly by pouring liquid nitrogen until the temperature went down to $77 \mathrm{~K}$, and opened the gap to $2 \mathrm{~mm}$. After that we inserted a Hall probe and held it at the center of the gap to measure the gap dependence of the magnetic field. In Fig. 4(b), the results of the measurements with and without the superconductors are shown by solid and dotted curves, respectively, which shows a clear enhancement of the magnetic field by the superconductor. As denoted by a chain curve, the current density in the superconductor is estimated from the field enhancement. As the gap was opened, the current density approached the $J_{c}$ value of $200 \mathrm{~A} / \mathrm{mm}^{2}$ that has been determined in the previous measurement. It should be noted, however, that the current density decreased at the gap wider than $8 \mathrm{~mm}$, which indicates that $J_{c}$ was decreased during the experiment. This performance degradation of the superconductor is probably due to a mechanical damage, i.e., increase in the amount of cracking.

\section{DISCUSSION}

The above experimental results strongly support the principle of $\mathrm{CU}$ plus as a scheme to enhance the field of the cryoundulator; however, there are two important performances to be improved in the superconductors that we used in the experiments. First, the mechanical stability should be improved to ensure a good reproducibility of the magnetic field, which is indispensable to a practical use as an undulator. In fact, several solutions have already been reported [16,17], which were not applied to our superconductor samples because of lack of time.

Second, superconductors with higher $J_{c}$ value are necessary because the $J_{c}$ value of the superconductor used in the experiments, $200 \mathrm{~A} / \mathrm{mm}^{2}$, is not enough to actively adopt the principle of CU plus, as is found from Fig. 2(c). It should be noted, however, that this is the value at the temperature of liquid nitrogen, i.e., $77 \mathrm{~K}$. It is well known that $J_{c}$ of a SC material has a strong dependence on the temperature $(T)$. In general, the relation between $J_{c}$ and $T$ is roughly expressed as[24]

$$
J_{c}(T)=J_{0}\left[1-\left(\frac{T}{T_{c}}\right)^{2}\right]^{m},
$$

where $J_{0}$ is the critical current density at $0 \mathrm{~K}, T_{c}$ the critical temperature, and the exponent $m$ is a parameter ranging from 1 to 3 which denotes a pinning mechanism of the type II superconductor. As an example, let us calculate $J_{c}$ at two temperatures of 77 and $40 \mathrm{~K}$ for $\mathrm{Gd}-$ $\mathrm{Ba}-\mathrm{Cu}-\mathrm{O}$ superconductor with $T_{c}=90 \mathrm{~K}$ and $m=2$. Then we find that $J_{c}(40 \mathrm{~K}) / J_{c}(77 \mathrm{~K}) \sim 9$. Accordingly, we can expect $J_{c}$ to be about $1.8 \mathrm{kA} / \mathrm{mm}^{2}$ at $40 \mathrm{~K}$ for the superconductor used in the experiment. In such a case, $B_{p}$ of CU plus is expected to be $1.45 \mathrm{~T}$ at the gap of $5 \mathrm{~mm}$, slightly higher than the $B_{p}$ value currently achieved in the conventional SCU. In addition, we emphasize that the performance of the copper-oxide SC material and related technologies are being improved year by year [15-19]. It is therefore expected that $\mathrm{SC}$ materials with enough $J_{c}$ to be used in CU plus will be, or have been already, developed. Thus, the concept of CU plus has a large possibility toward shorter period undulator development.

[1] D. Attwood, K. Halbach, and K. J. Kim, Science 228, 1265 (1985).

[2] J. Chavanne, P. Elleaume, and P.V. Vaerenbergh, J. Synchrotron Radiat. 5, 196 (1998).

[3] E. Gluskin, J. Synchrotron Radiat. 5, 189 (1998).

[4] H. Kitamura, T. Bizen, T. Hara, X. Maréchal, T. Seike, and T. Tanaka, Nucl. Instrum. Methods Phys. Res., Sect. A 467-468, 110 (2001). 
[5] P. M. Stefan, T. Tanabe, S. Krinsky, G. Rakowsky, L. Solomon, and H. Kitamura, J. Synchrotron Radiat. 5, 417 (1998).

[6] T. Schmidt, G. Ingold, A. Imhof, B. D. Patterson, L. Patthey, C. Quitmann, C. Schulze-Briese, and R. Abela, Nucl. Instrum. Methods Phys. Res., Sect. A 467-468, 126 (2001) .

[7] N.V. Smith, Radiat. Phys. Chem. 70, 641 (2004).

[8] H. Kitamura, J. Synchrotron Radiat. 7, 121 (2000).

[9] S. Yamamoto, T. Shioya, M. Hara, H. Kitamura, X.W. Zhang, T. Mochizuki, H. Sugiyama, and M. Ando, Rev. Sci. Instrum. 63, 400 (1992).

[10] T. Hara, T. Tanaka, T. Tanabe, X. Maréchal, S. Okada, and H. Kitamura, J. Synchrotron Radiat. 5, 403 (1998).

[11] M. Bazin, Y. Farge, M. Lemonnier, J. Perot, and Y. Petroff, Nucl. Instrum. Methods 172, 61 (1980)

[12] S. Chouhan, R. Rossmanith, S. Strohmer, D. Doelling, A. Geisler, A. Hobl, and S. Kubsky, in Proceedings of the Particle Accelerator Conference (PAC 2003), Portland, OR, 2003 (IEEE, Piscataway, NJ, 2003).

[13] T. Hara, T. Tanaka, H. Kitamura, T. Bizen, T. Seike, T. Kohda, and Y. Matsuura, Phys. Rev. ST Accel. Beams 7, 050702 (2004).

[14] T. Bizen, T. Asano, T. Hara, X. Maréchal, T. Seike, T. Tanaka, H. Kitamura, H.S. Lee, and D. E. Kim, in Eighth International Conference on Synchrotron Radiation Instrumentation, edited by T. Warwick, J. Arthur, H. A. Padmore, and J. Stöhr, AIP Conf. Proc. No. 705 (AIP, New York, 2004), pp. 167-170.

[15] S. Nariki, N. Sakai, and M. Murakami, Physica (Amsterdam) 341C-348C, 2409 (2000).

[16] M. Tomita, M. Murakami, K. Sawa, and Y. Tachi, Physica (Amsterdam) 357C-360C, 690 (2001)

[17] M. Tomita and M. Murakami, Nature (London) 421, 517 (2003).

[18] H. Ikuta, S. Ikeda, A. Mase, M. Yoshikawa, Y. Yanagi, Y. Itoh, T. Oka, and U. Mizutani, Appl. Supercond. 6, 109 (1998).

[19] M. Muralidhar, N. Sakai, M. Jirsa, M. Murakami, and N. Koshizuka, Supercond. Sci. Technol. 16, L46 (2003).

[20] T. Kohda (private communication).

[21] O. Chubar, P. Elleaume, and J. Chavanne,J. Synchrotron Radiat. 5, 481 (1998).

[22] R. Rossmanith, H. Moser, A. Geisler, A. Hobl, D. Krischel, and M. Schillo, in Proceedings of the European Particle Accelerator Conference (EPAC 2002), Paris, 2002 (EPS-IGA/CERN, Geneva, 2002), p. 2628.

[23] K. Halbach, J. Phys. C 1, 211 (1983).

[24] Z. Koziol, J. J. M. Franse, P. F. de Chatel, and A. A. Menovsky, Phys. Rev. B 50, 15978 (1994) 